\title{
Un sistema ultrasónico de bajo costo para la localización de equipo oceanográfico en aguas someras
}

\author{
A Low-Cost Ultrasonic System for Locating Oceanographic \\ Equipment in Shallow Water
}

\author{
Hernández-Reyes Alberto Isaac \\ Instituto Politécnico Nacional \\ Centro de Investigación en Ciencia Aplicada y Tecnología Avanzada \\ Unidad Altamira, Tamaulipas \\ Correo: isaac.h.r.86@gmail.com \\ Ulloa Marco Julio \\ Instituto Politécnico Nacional \\ Centro de Investigación en Ciencia Aplicada y Tecnología Avanzada \\ Unidad Altamira, Tamaulipas \\ Correo: sidescansonar2003@yahoo.com.mx \\ Ortega-Izaguirre Rogelio \\ Instituto Politécnico Nacional \\ Centro de Investigación en Ciencia Aplicada y Tecnología Avanzada \\ Unidad Altamira, Tamaulipas \\ Correo:acroi@hotmail.com
}

Información del artículo: recibido: febrero de 2016, reevaluado: marzo de 2016, aceptado: mayo de 2016

\section{Resumen}

En el presente trabajo se describen las características más importantes del primer prototipo de un sistema ultrasónico capaz de detectar frecuencias de 26 $\mathrm{kHz}$ en profundidades ligeramente mayores de $30 \mathrm{~m}$. El sistema surge de la necesidad de apoyar los trabajos de localización y recuperación de equipo oceanográfico que se fondea sin boya de señalización superficial en aguas someras. Los elementos que componen el sistema son: un circuito electrónico de transmisión y recepción de señales acústicas, un transductor acústico piezoeléctrico y una carcasa resistente a la presión. La electrónica del transceptor contiene un algoritmo que calcula la distancia entre un par de transceptores con base en el tiempo de propagación de la señal ultrasónica. La carcasa está hecha de PVC, un material de bajo costo y fácil de mecanizar. Los resultados de las pruebas operativas indican un error cuadrático medio de $\pm 0.4 \mathrm{~m}$ para la distancia entre transceptores, que es un orden de magnitud menor al error de precisión de ubicación en los sistemas de posicionamiento global. El sistema ultrasónico constituye una herramienta de apoyo idónea para disminuir el riesgo de pérdida de equipo oceanográfico por eventos hidrometeorológicos o actividades pesqueras en cuerpos de agua someros.

\section{Descriptores:}

- transceptor acústico

- anclaje en aguas someras

- oceanografía costera 


\begin{abstract}
This paper describes the most important features of the first prototype of an ultrasonic system capable of detecting frequencies of $26 \mathrm{kHz}$ in depths slightly greater than $30 \mathrm{~m}$. The system arises from the need to support the fieldwork of locating and recovering oceanographic equipment moored without surface buoy in shallow waters. The elements of the system are: an electronic circuit for transmitting and receiving acoustic signals, a piezoelectric acoustic transducer and a pressure resistant housing. The electronics in the transceiver contains an algorithm that calculates the distance between a pair of transceivers based on the propagation time of the ultrasonic signal. Housing is made of PVC, a material inexpensive and easy to machine. The results of operational tests indicate a root mean square of $\pm 0.4 \mathrm{~m}$ for the distance between transceivers, being an order of magnitude less than the precision error location in global positioning systems. The ultrasonic system is an ideal support tool to reduce the risk of loss of oceanographic equipment caused by hydrometeorological events or fishing activities in shallow water bodies.
\end{abstract}

\section{Introducción}

La ingeniería electrónica es fundamental para obtener mediciones confiables de las corrientes, las olas y las propiedades del agua en los cuerpos marinos, salobres y de agua dulce. Las aplicaciones son amplias, pues incluyen el desarrollo de tecnologías para la utilización de fuentes de energía renovable oceánicas, el diseño y construcción de instrumentos para estudios del manejo integral y desarrollo sustentable de los ecosistemas costeros y para la búsqueda de evidencia de contaminación por hidrocarburos en aguas profundas a través de vehículos autónomos submarinos, así como para la dispersión de contaminantes en aguas superficiales, entre otros. Una gran diversidad de instrumentos y carcasas para proteger los sistemas embebidos se han diseñado de acuerdo con el fenómeno o variable a medir, al método de medición, y también de acuerdo con las condiciones ambientales adversas a resistir (e.g. Chereskin y Howe, 2007; Ulloa, 2007; Vera, 2007; Joseph, 2014).

La instrumentación electrónica, como los correntómetros y las sondas multiparámetros por mencionar algunos, puede dividirse de acuerdo con el método de medición en (Chereskin y Howe, 2007): puntual (anclaje, lance, sondeo), lagrangiana (boya de deriva, dispersión de colorante) y remota (acústica, electromagnética). De particular interés son los instrumentos acústicos que se fondean en un punto fijo cerca de las ciudades costeras, bocanas de lagunas y las desembocaduras de los ríos, pues suelen presentarse dificultades durante las labores de localización/recuperación, entre las cuales se encuentra la turbiedad del agua por el efecto de las corrientes y el oleaje. Si la visibilidad es insuficiente, puede ocurrir que el buzo no localice el instrumento o que le lleve demasiado tiempo encontrarlo. Además de colocar sedimentos en suspensión que pueden cubrir el equipo de medición, las corrientes y el oleaje poseen la capacidad de mover el equipo fuera de su posición original, lo que complica el trabajo de localización/recuperación. Una solución consiste en señalizar la posición de fondeo mediante una boya superficial o subsuperficial (e.g. Trask y Weller, 2001), sin embargo, resulta inconveniente porque el instrumento queda a merced de la curiosidad de los pescadores locales, así como del arrastre de las redes que se utilizan durante la captura de camarón (e.g. INP, 2000). Otra solución es colocar en el instrumento un mecanismo de liberación acústico (Joseph, 2014), de tal modo, que al estar unido a una boya subsuperficial, pueda ascender por flotabilidad y recuperarse en la superficie del agua. No obstante, dicha solución es impráctica, pues se pierde la plataforma de trípode donde se monta el instrumento (anclaje), además de que en caso de enterramiento es probable que la boya no proporcione flotabilidad suficiente para recuperarlos. Aunque la ubicación del instrumento se determina por medio de un sistema de posicionamiento global, es mejor contar con un dispositivo auxiliar que apoye la labor de localización/recuperación del anclaje en profundidades del orden de $30 \mathrm{~m}$. El objetivo de este trabajo consistió en diseñar y construir el prototipo de un sistema ultrasónico de bajo costo para localizar instrumentos fondeados en aguas someras. 


\section{Desarrollo}

\section{Diseño del sistema}

El sistema ultrasónico que se diseñó y construyó consta de un par de transceptores. Básicamente, un transceptor consta de un transductor acústico piezoeléctrico (e.g. Kleeman y Kuc, 2008), un circuito electrónico, las baterías y la carcasa. El circuito electrónico se compone de un microcontrolador Atmel AVR, un transmisor, un receptor y la fuente de alimentación (figura 1). En el microcontrolador se generan señales ultrasónicas con una frecuencia de $(26 \pm 1) \mathrm{kHz}$ mediante un algoritmo (Hernández, 2015). Adicionalmente, se agregó un relevador mecánico con bobina de $5 \mathrm{~V}$ a fin de conmutar el sensor piezoeléctrico con el transmisor de potencia durante el tiempo que se transmite la señal ultrasónica, así como al receptor durante el tiempo de espera. En este trabajo se empleó una batería recargable sellada de ácido-plomo, de $12 \mathrm{~V}$ con capacidad de $1.2 \mathrm{~A} / \mathrm{h}$ para la alimentación de los circuitos. La tabla 1 resume las características del circuito electrónico.

Tabla 1. Especificaciones del circuito electrónico

\begin{tabular}{lc}
\hline \multicolumn{1}{c}{ Descripción } & Especificación \\
\hline Tamaño del circuito & $10 \times 8 \mathrm{~cm}$ \\
Voltaje a la salida del regulador de voltaje & $4.97 \mathrm{~V}$ \\
Corriente eléctrica consumida sin transmisión & $0.002 \mathrm{~A}$ \\
Corriente eléctrica consumida con transmisión & $0.117 \mathrm{~A}$ \\
Duración de la batería con transmisión & $10 \mathrm{~h}$ \\
Duración de la batería sin transmisión & $25 \mathrm{~d}$ \\
\hline
\end{tabular}

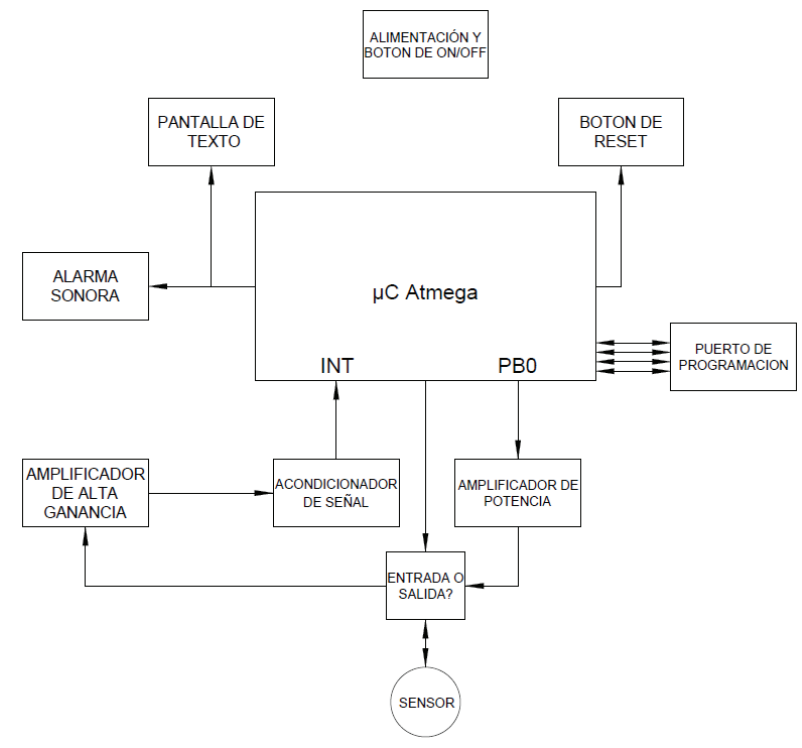

Figura 1. Descripción ilustrada del circuito electrónico del transceptor acústico
Uno de los transceptores, denominado el transceptor submarino (TS), se fondea junto con el anclaje donde permanece en un modo pasivo continuo (modo de espera). El otro transceptor, denominado el transceptor de rastreo (TR), se utiliza para estimar la distancia a la que se encuentra el transceptor submarino, lo cual implica ubicarse cerca de la posición del anclaje a bordo de una embarcación menor. Para la comunicación entre los transceptores TS y TR, se desarrolló el siguiente protocolo de aplicación para la transmisión-recepción. El TR emite una señal ultrasónica que se forma por una ráfaga de pulsos con una duración de $1.02 \mathrm{~s}$ y espera $9.18 \mathrm{~s}$ para recibir una señal de respuesta por parte del TS. En cuanto el TS recibe y detecta los pulsos a la frecuencia de emisión, se espera un tiempo muerto de $2 \mathrm{~s}$ para que lleguen todos los pulsos que transmitió el TR. Luego, el TS emite una primera señal de respuesta al TR que consiste en una ráfaga de pulsos con una duración de $1.02 \mathrm{~s}$ y espera $7.18 \mathrm{~s}$, la diferencia entre $9.18 \mathrm{~s}$ y $2 \mathrm{~s}$, para después emitir una segunda serie de pulsos y de ahí pasar nuevamente al modo de espera. Cuando el TR recibe la primera señal de respuesta, espera un tiempo de $2 \mathrm{~s}$ para que lleguen todos los pulsos del TS y queda en modo de espera de la segunda ráfaga de pulsos del TS. Este proceso se repite indefinidamente.

El circuito del TR mide el tiempo desde que se emite la señal ultrasónica hasta que recibe un par de señales de respuesta por parte del TS, el cual se denomina aquí tiempo medido $\left(t_{m}\right)$. El tiempo que se almacena en la memoria del microcontrolador corresponde a la suma de un ciclo del TR (10.20 s) en tres intervalos de viaje de la señal ultrasónica entre el TR y el TS, y el $\left(t_{m}\right)$. Existen dos tiempos que no se contabilizan en el microcontrolador, a saber, el tiempo de espera del TR de $2 \mathrm{~s}$, así como un tiempo muerto de $0.25 \mathrm{~s}$ que corresponde a una medida cualitativa del tiempo de respuesta de los circuitos electrónicos inherentes en el microcontrolador (Hernández, 2015). Entonces, la distancia entre transceptores (d) se estima como

$d=c\left[\frac{\left|t_{m}+2-10.2\right|-0.25}{3}\right]$

La velocidad del sonido en el océano, $c$, se considera constante $(1500 \mathrm{~m} / \mathrm{s})$.

\section{Circuito electrónico}

La figura 2 muestra el diagrama electrónico del circuito, correspondiente a los bloques en la figura 1. El circuito electrónico contiene el microcontrolador AVR de 8 bits, 
ATmega32, que cuenta con las siguientes características: alto rendimiento y bajo consumo de energía, $1 \mathrm{~kb}$ de EEPROM, un convertidor A/D de 10 bit, así como arquitectura avanzada RISC con 131 instrucciones en lenguaje ensamblador. Además, opera en el intervalo 4.5-5.5 V, su consumo de energía es de $1.1 \mathrm{~mA}$ cuando está activo y $0.35 \mathrm{~mA}$ en modo inactivo. El microcontrolador opera con un reloj interno de $8 \mathrm{MHz}$. Los requerimientos que la electrónica debe satisfacer los impone el fabricante. Con el objeto de generar la onda acústica que se aplica al transmisor, se implementaron un conjunto de instrucciones en el entorno del programa Bascom AVR®. La ejecución de las instrucciones genera una señal cuadrada (TTL) en el pin PB0 del microcontrolador.

El receptor se compone de dos etapas: amplificador de señal y acondicionador de señal. En la primera etapa, la señal que recibe el sensor receptor se amplifica 1000 veces en dos pasos, mediante un amplificador por 100 y un amplificador por 10, tal como se observa en la figura 2. Cada paso se refiere a la relación establecida por las resistencias de retroalimentación mediante configuración inversora. Como estos dos pasos son en cascada, ambas ganancias se multiplican y de ahí una ganancia de 1000. En estas etapas de amplificación se utilizó un circuito integrado LM358; sus características de acuerdo con el fabricante son: alta ganancia en volta$\mathrm{je}, 10^{5}$, frecuencia compensada en temperatura, frecuencia máxima de $1.1 \mathrm{MHz}$, operación con fuente simple y con voltaje de alimentación 3-32 V dc, bajo offset $2 \mathrm{mV}$, temperatura $(0-70){ }^{\circ} \mathrm{C}$ y encapsulados DIP8, SO8 Mini $\mathrm{SO} 8$ y TSSOP8. Hasta este punto, la forma de onda de la señal recibida (TS) se muestra en la figura 3a.

Designando la alta ganancia en voltaje del circuito LM358 como $A_{0}=105$, donde $\beta$ es el factor de realimentación establecido por las resistencias y de acuerdo con el diagrama del circuito electrónico de la figura 2, se tiene que en el primer paso de amplificación, la ganancia finita del circuito se da por, $v_{0} / v_{i}=\left(\beta+1 / A_{0}\right)^{-1}=100.89$, donde $v_{0}$ $\mathrm{y} v_{i}$ son los voltajes de salida y de entrada al circuito amplificador, respectivamente, $y \beta=101^{-1}$. El error relativo debido a esta ganancia finita del amplificador operacional es $1 / 1000$ o $0.1 \%$. El error relativo correspondiente al segundo paso de amplificación es $0.01 \%$.

En la segunda etapa, la señal amplificada se acondiciona en un circuito que consiste de un diodo y un comparador (figura 2). El acondicionador de señal cumple el rol de convertir toda la ráfaga de pulsos que se recibe en un pulso único. Este pulso único es el que recibe la entrada de interrupción del microcontrolador. El criterio para verificar el tren de pulsos acústicos es que los conos de

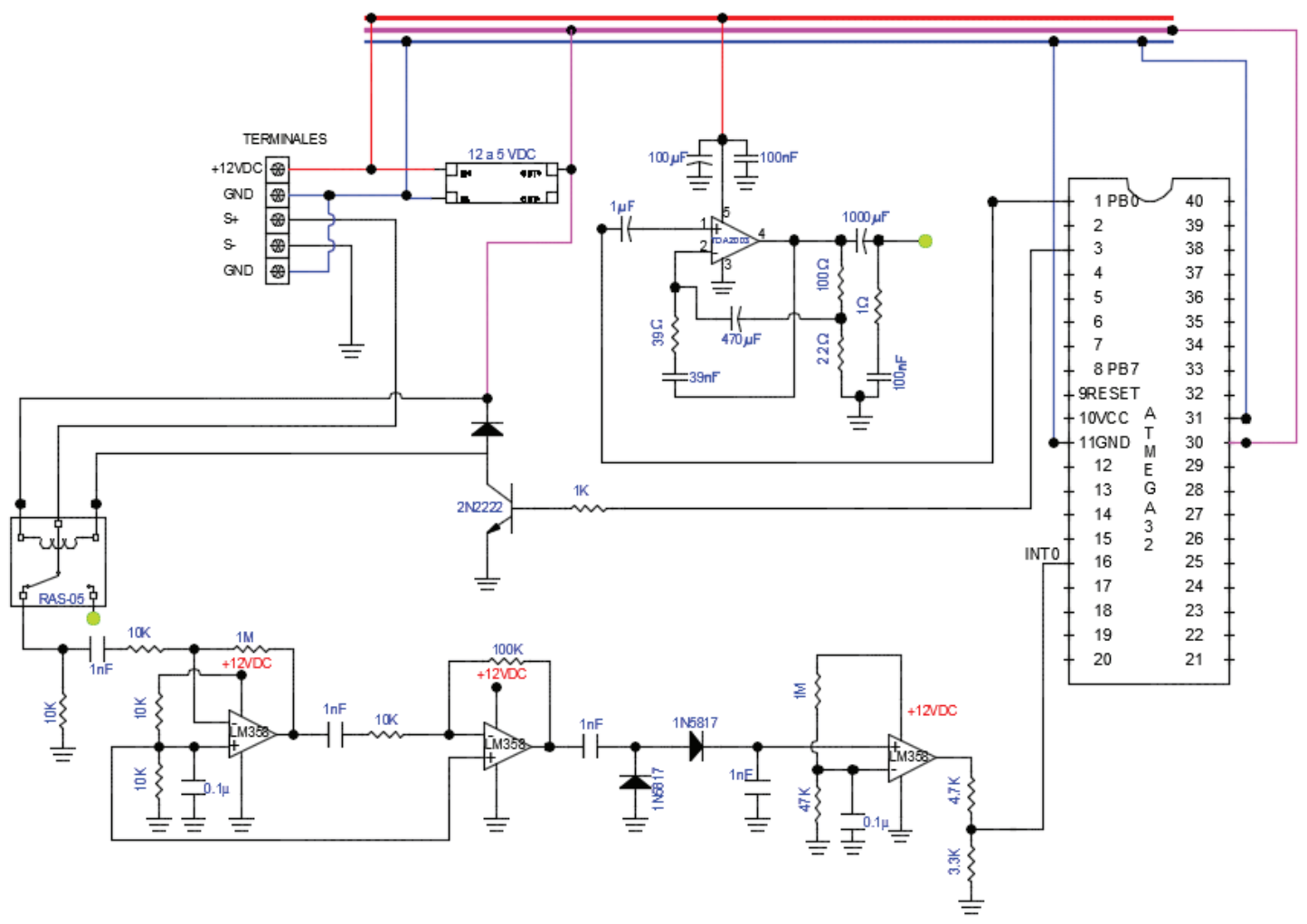

Figura 2. Diagrama electrónico simplificado del circuito de la figura 1 

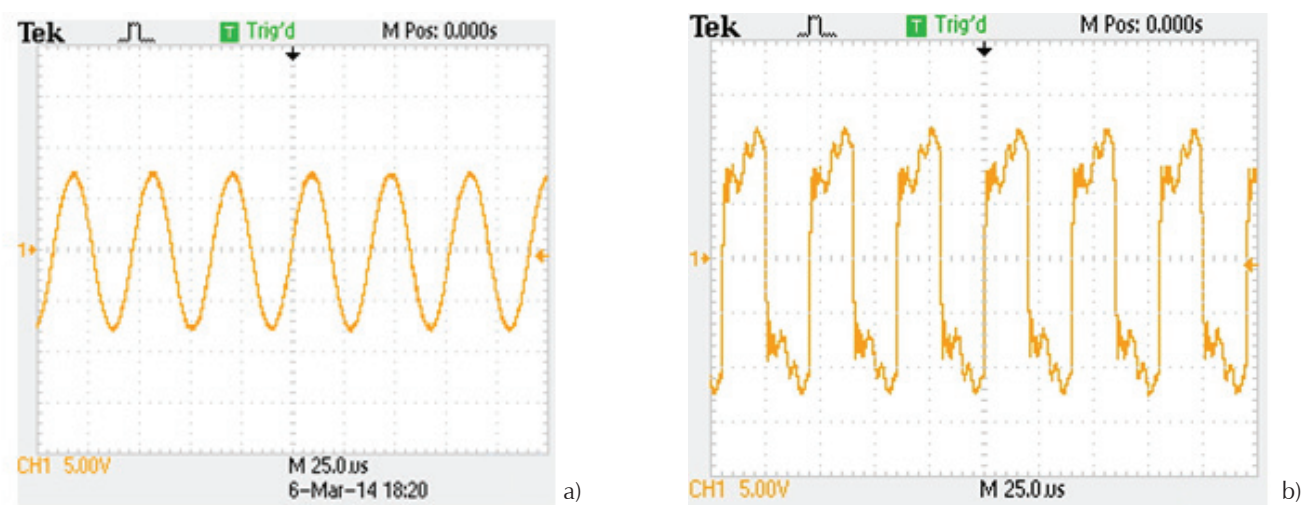

Figura 3. a) onda que recibe el TS, b) onda que transmite el TR

transmisión/recepción se encuentren alineados lo más posible, pues así los transceptores pueden sincronizarse para calcular la distancia entre ellos. En cuerpos de agua someros, la profundidad mínima en la cual puede operar una embarcación menor es de unos $0.5 \mathrm{~m}$, en tanto que la altura de la plataforma trípode donde se coloca equipo de medición acústico es aproximadamente de $0.5 \mathrm{~m}$. Una profundidad de fondeo segura para evitar que la embarcación colisione con el equipo sería de unos $2 \mathrm{~m}$. Es por ello que se considera que la aplicación práctica del sistema ultrasónico en el campo no requiere algoritmo anticolisión para equipo de medición fondeado en profundidades mayores de $2 \mathrm{~m}$.

El transmisor de potencia consta de un circuito integrado TDA2003 que viene en un encapsulado llamado Pentawatt. Dicho encapsulado está diseñado para funcionar con una alimentación simple desde $8 \mathrm{~V}$ hasta $18 \mathrm{~V}$. Los componentes que se requieren para su buen funcionamiento se detallan en la hoja que proporciona el fabricante. La figura $3 b$ ilustra la forma de la onda que se transmite (TR). El voltaje máximo de salida, que corresponde a la señal acústica que se transmite, es de $9 \mathrm{~V}$ pico.

\section{Carcasa}

La presión es la variable oceanográfica que determina la geometría de las carcasas de la instrumentación electrónica, por lo general cilindros o esferas (Chereskin y Howe, 2007; Joseph, 2014; MacDonald y Mullarney, 2015). Las carcasas protegen las partes internas de los transceptores (los transductores y toda la electrónica) cuando se colocan bajo el mar o en cuerpos de agua dulceacuícolas. Los materiales que componen las carcasas deben resistir o protegerse de la corrosión marina. En profundidades menores de $600 \mathrm{~m}$, es frecuente utilizar materiales plásticos (Chereskin y Howe, 2007), por ejemplo, PVC (Joseph, 2014; Murphy et al., 2015).
La figura 4 muestra la carcasa cilíndrica que se diseñó para alojar los transductores. Se eligió PVC como material de construcción por su bajo costo, disponibilidad y facilidad de mecanizado en comparación con titanio o delrin ${ }^{\circledR}$. La carcasa se compone de un tapón roscado hembra cedula 40 (6 $\mathrm{mm}$ de espesor) y de diámetro nominal de $100 \mathrm{~mm}$, un adaptador espiga roscado macho cedula 40 (6 $\mathrm{mm}$ de espesor) y de diámetro nominal de $100 \mathrm{~mm}$, un tramo de tubo de $100 \mathrm{~mm}$ de diámetro cuya longitud puede variar según se requiera, y una tapa ciega. El diseño lo completa una rueda de acrílico de $6 \mathrm{~mm}$ de espesor que constituye la ventana acústica, y sobre la cual se fija el transductor piezoeléctrico. La carcasa puede resistir presiones hasta de 413 $686.6 \mathrm{~Pa}$ (60 psi o 4 atm) antes que la rueda de acrílico se fracture de acuerdo con las pruebas de estanqueidad realizadas por Hernández (2015). La figura 5 muestra una fotografía del transceptor submarino. El TS se monta sobre la plataforma de trípode que sujeta también al equipo de medición. La plataforma se baja al fondo desde una embarcación mediante un cabo y con la ayuda de buzos.

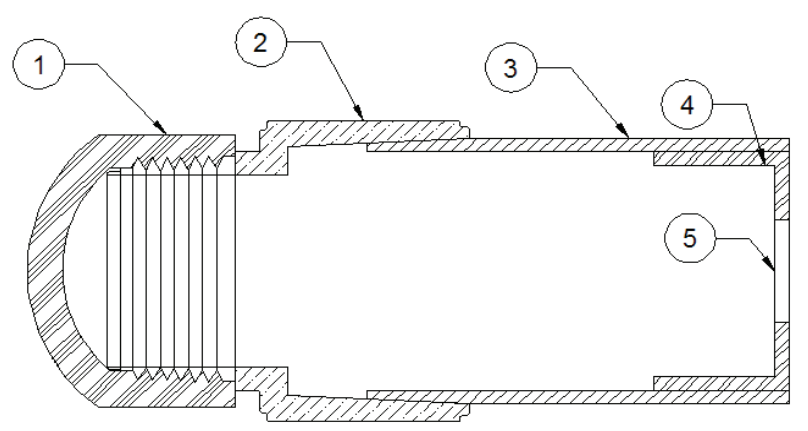

Figura 4. Descripción ilustrada de la carcasa, 1) tapón roscado hembra, 2) adaptador espiga roscado macho, 3) tramo de tubo, 4) tapa ciega, 5) rueda de acrílico 


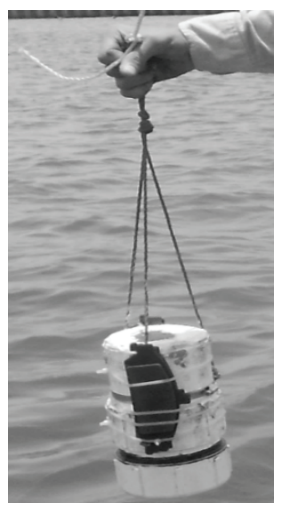

Figura 5. El transceptor submarino con pesos sujetos a la carcasa mediante cinchos a fin de hundirlo en el agua y realizar pruebas de campo. El transceptor de rastreo tiene la misma forma

\section{Resultados y discusión}

Se realizaron dos pruebas del funcionamiento del sistema ultrasónico en Playa Miramar, frente a la costa de Ciudad Madero, Tamaulipas (22 $16^{\prime} 20.40^{\prime \prime} \mathrm{N}, 9^{\circ} 44^{\prime}$ $4.46^{\prime \prime} \mathrm{O} ; 22^{\circ} 16^{\prime} 55.61^{\prime \prime} \mathrm{N}, 97^{\circ} 42^{\prime} 0.07^{\prime \prime} \mathrm{O}$ ), a una profundidad de 20 y $33 \mathrm{~m}$, respectivamente. En estos dos sitios se midió el perfil de la velocidad del sonido con un equipo denominado CastAway ${ }^{\mathrm{TM}}$-CTD (YSI, 2010). Cada prueba consistió en colocar el TR sobre la superficie del agua a bordo de una embarcación menor con motor fuera de borda anclada al fondo y orientada en dirección de la corriente promedio, para luego bajar gradualmente el TS en profundidades conocidas. En cada profundidad se efectuaron cinco mediciones y se estimó la distancia al TR. El promedio de estas se muestra en las figuras $6 a$ y 7 a con sus respectivas desviaciones estándar, y los valores correspondientes en la tabla 2.

Las variaciones en las desviaciones estándar (figuras 6a y 7a) sugieren movimientos verticales a causa del oleaje y el efecto advectivo del flujo superficial promedio, así como variaciones en el alineamiento de los conos de transmisión entre el TS y el TR dentro del área de emisión ultrasónica. En ausencia de olas, dicha área es del orden de $300 \mathrm{~m}^{2}$ a una profundidad de $30 \mathrm{~m}$. Las ubicaciones de los puntos estuvieron razonablemente lejos de la influencia de la corriente de descarga del río Pánuco.

En los primeros $3 \mathrm{~m}$ de profundidad, el perfil de la velocidad del sonido muestra la influencia dominante de la variación vertical de la salinidad sobre la variación correspondiente a la temperatura (figuras $6 \mathrm{~b}$ y $7 \mathrm{~b}$ ). En el resto de la columna de agua, los cambios que se observan en la velocidad del sonido se deben principalmente a las variaciones verticales de la temperatura. $\mathrm{La}$ velocidad del sonido en la columna de agua (figuras $6 \mathrm{~b}$ y $7 b$ ) fue mayor al valor nominal del océano y, por ello, se utilizaron valores promedio locales para el cálculo de la distancia entre transceptores. Las velocidades del sonido promedio a $20 \mathrm{~m}$ y $33 \mathrm{~m}$ son en orden respectivo, $1530.5 \mathrm{~m} / \mathrm{s}$ y $1530.0 \mathrm{~m} / \mathrm{s}$, con una variación medida en el campo de $\pm 4.7 \mathrm{~m} / \mathrm{s} \mathrm{y} \pm 4.1 \mathrm{~m} / \mathrm{s}$, respectivamente.

El valor del error cuadrático medio de las distancias entre transceptores, evaluado como:
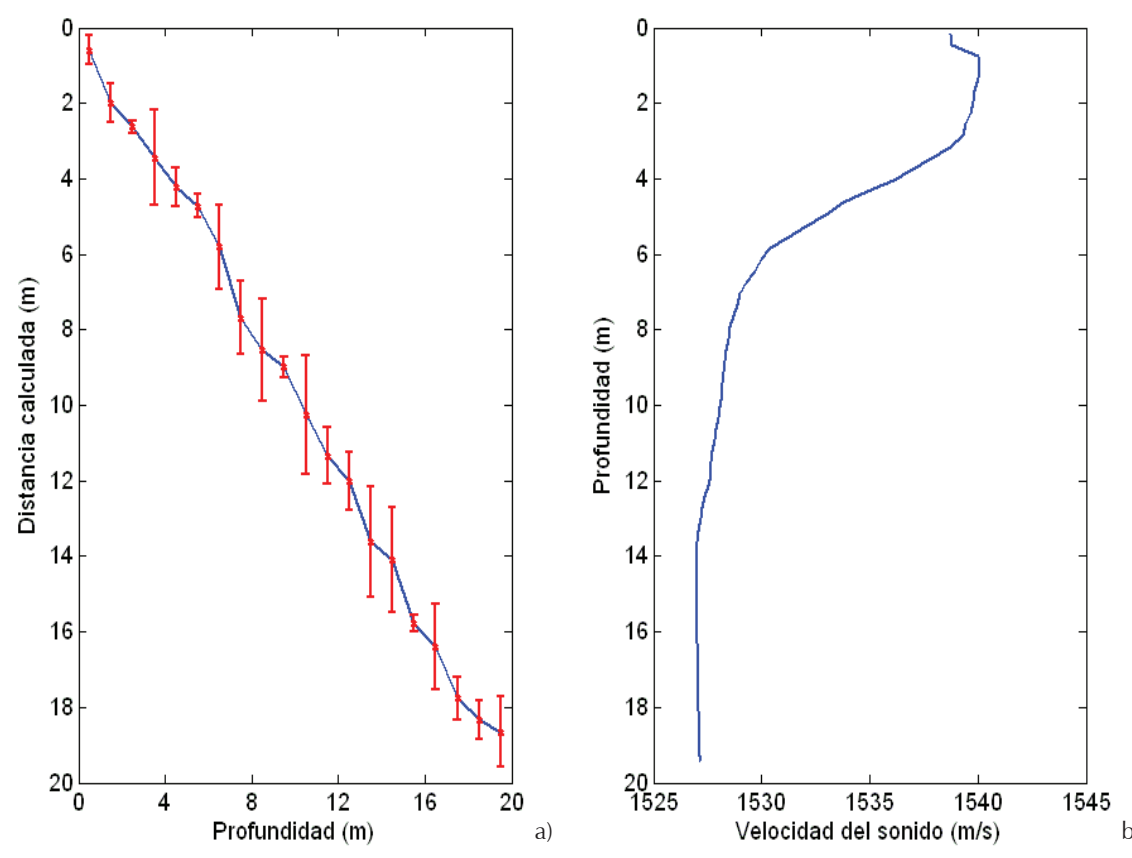

Figura 6. a) Distancias entre transceptores en función de la profundidad, b) perfil de la velocidad del sonido. Mediciones realizadas en la posición $22^{\circ} 16^{\prime} 20.40^{\prime \prime}$ de latitud norte y $97^{\circ} 44^{\prime} 4.46^{\prime \prime}$ de longitud oeste 

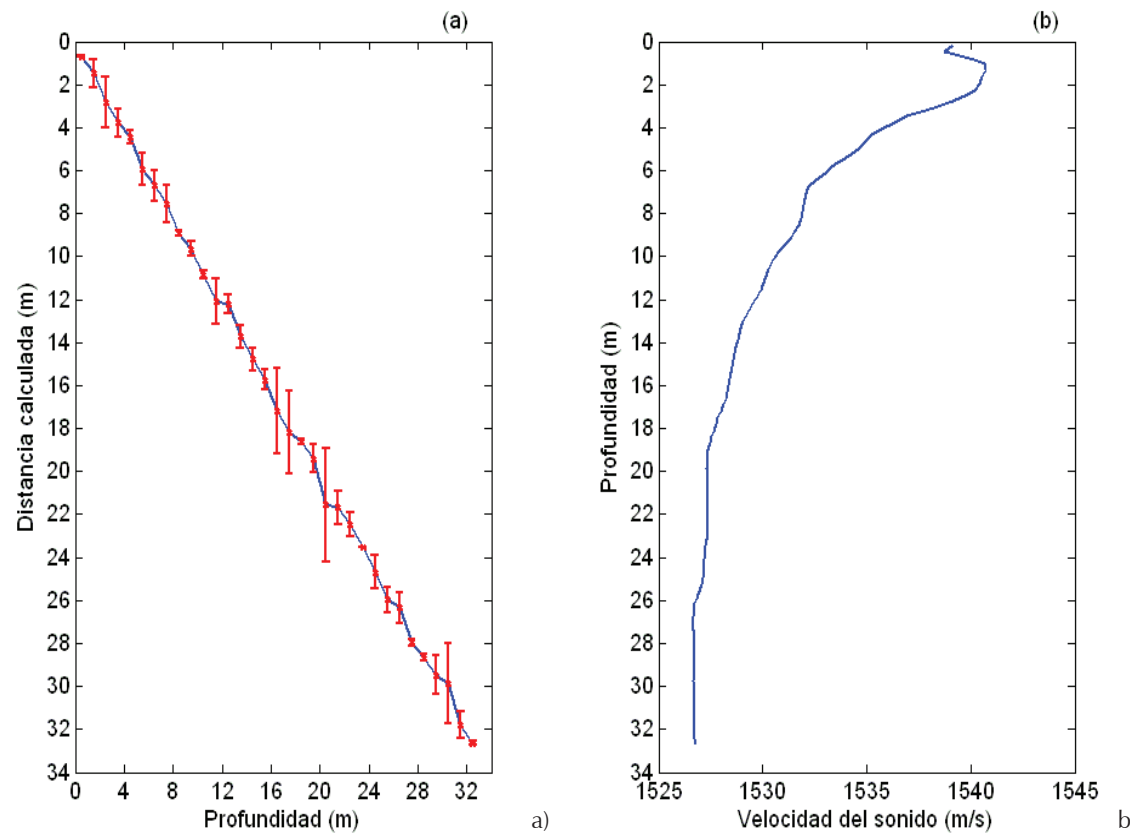

Figura 7. a) Distancias entre transceptores en función de la profundidad, b) perfil de la velocidad del sonido. Mediciones realizadas en la posición $22^{\circ} 16^{\prime} 55.61^{\prime \prime}$ de latitud norte y $97^{\circ} 42^{\prime} 0.07^{\prime \prime}$ de longitud oeste

Tabla 2. Distancias medidas entre el TR y el TS a intervalos de profundidad de $1 \mathrm{~m}$

\begin{tabular}{|c|c|c|c|c|}
\hline Profundidad (m) & Distancia (m) & Desviación estándar (m) & Distancia (m) & Desviación estándar (m) \\
\hline 0.5 & 0.6 & 0.4 & 0.7 & 0.0 \\
\hline 1.5 & 2.0 & 0.5 & 1.5 & 0.6 \\
\hline 2.5 & 2.6 & 0.2 & 2.8 & 1.2 \\
\hline 3.5 & 3.4 & 1.3 & 3.8 & 0.7 \\
\hline 4.5 & 4.2 & 0.5 & 4.4 & 0.3 \\
\hline 5.5 & 4.7 & 0.3 & 5.9 & 0.8 \\
\hline 6.5 & 5.8 & 1.1 & 6.7 & 0.7 \\
\hline 7.5 & 7.7 & 1.0 & 7.6 & 0.8 \\
\hline 8.5 & 8.5 & 1.3 & 8.9 & 0.1 \\
\hline 9.5 & 9.0 & 0.3 & 9.6 & 0.3 \\
\hline 10.5 & 10.2 & 1.6 & 10.9 & 0.2 \\
\hline 11.5 & 11.3 & 0.7 & 12.1 & 1.0 \\
\hline 12.5 & 12.0 & 0.8 & 12.2 & 0.4 \\
\hline 13.5 & 13.6 & 1.5 & 13.7 & 0.5 \\
\hline 14.5 & 14.1 & 1.4 & 14.8 & 0.5 \\
\hline 15.5 & 15.8 & 0.2 & 15.7 & 0.4 \\
\hline 16.5 & 16.4 & 1.1 & 17.2 & 2.0 \\
\hline 17.5 & 17.8 & 0.6 & 18.2 & 1.9 \\
\hline 18.5 & 18.3 & 0.5 & 18.6 & 0.1 \\
\hline 19.5 & 18.6 & 0.9 & 19.4 & 0.6 \\
\hline 20.5 & & & 21.6 & 2.7 \\
\hline 21.5 & & & 21.7 & 0.8 \\
\hline 22.5 & & & 22.5 & 0.6 \\
\hline 23.5 & & & 23.5 & 0.0 \\
\hline 24.5 & & & 24.7 & 0.8 \\
\hline 25.5 & & & 26.0 & 0.6 \\
\hline 26.5 & & & 26.3 & 0.7 \\
\hline 27.5 & & & 27.9 & 0.1 \\
\hline 28.5 & & & 28.7 & 0.1 \\
\hline 29.5 & & & 29.5 & 0.9 \\
\hline 30.5 & & & 29.9 & 1.9 \\
\hline 31.5 & & & 31.8 & 0.6 \\
\hline 32.5 & & & 32.6 & 0.1 \\
\hline
\end{tabular}




$$
\left[\frac{\sum_{i=1}^{n}\left(p_{i}-d_{i}\right)^{2}}{n}\right]^{\frac{1}{2}}
$$

donde

$p=$ profundidad

$d=$ distancia calculada por el TR y

$n$ = número de mediciones

fue de $\pm 0.40 \mathrm{~m}$ en una columna de agua con un tirante de aproximadamente $20 \mathrm{~m}$ (figura $6 \mathrm{a}$ ) y de $\pm 0.37 \mathrm{~m}$ en una columna de agua de unos $33 \mathrm{~m}$ de profundidad (figura 7a). Estos errores son inherentes a la estimación de las profundidades, donde se situó el TS, pero son un orden de magnitud menor al error de precisión de ubicación en los sistemas de posicionamiento global con tecnología del Sistema de Aumentación de Área Amplia que es del orden de \pm (1-3)m (Madry, 2015).

Aunque en el cálculo de la distancia entre transceptores se consideró la velocidad del sonido constante, en realidad es una función de la temperatura, la salinidad, la presión estática y sus perfiles dependen de la localidad, la estación del año, del clima y los procesos de mezcla que se producen en la capa superficial del mar por la acción del viento y el oleaje (Waite, 2005). La propagación del sonido depende a su vez de las fronteras (superficie, fondo) y los dispersores acústicos presentes en la columna de agua, por ejemplo, burbujas de aire, sedimentos en suspensión y organismos (Medwin, 2005). Entonces, como los transceptores requieren tener previamente asignado un valor promedio constante de la velocidad del sonido, la localización de equipo oceanográfico en cualquier época del año dependerá de contar con una buena estimación local de la velocidad del sonido. Lo anterior se puede lograr con la caracterización anual de la velocidad del sonido en la zona de estudio donde se instalen anclajes. Por otra parte, la inhomogeneidad de la columna de agua produce una pérdida exponencial de la señal ultrasónica durante las etapas de transmisión y recepción, ocasionando a su vez que la amplitud de la señal sea altamente variable. Por ejemplo, para la señal acústica de $9 \mathrm{~V}$ pico que transmite el TR, el TS únicamente recibe voltajes del orden de cientos de $\mathrm{mV}$. Dicha pérdida no puede evitarse y es la razón por la cual se necesita, en el diseño de los transceptores, un circuito amplificador de señales.

En áreas costeras donde la actividad pesquera es activa, el equipo oceanográfico generalmente se fondea sin boyas superficiales que indiquen su ubicación. El prototipo del sistema ultrasónico constituye una buena herramienta de apoyo para la recuperación del equipo, pues permite posicionar la embarcación prácticamente sobre el anclaje de manera que el buzo lo encuentre con relativa rapidez, minimizando así el riesgo de pérdida cuando se haya movido fuera de su posición original a causa de la dinámica costera o de las actividades propias de la pesca de arrastre. El costo de los transceptores es aproximadamente $40 \%$ menor al costo de los sistemas comerciales, los cuales no son de manufactura nacional. El sistema actual tiene la ventaja de que el circuito receptor puede modificarse fácilmente para medir el nivel de ruido submarino, tema de interés en la evaluación de propuestas para la construcción de infraestructura en habitat marinos (Williams et al., 2005; Farcas et al., 2016). En términos de mejoras al prototipo, se requiere incrementar el rendimiento de la batería con el objeto que el sistema permanezca montado en anclajes oceanográficos someros por un periodo mayor de 30 días, así como disminuir el error cuadrático medio de las distancias entre transceptores. La carcasa, aunque funcional, no es estética por lo que una mejora al diseño y menores dimensiones es parte de un trabajo futuro.

\section{Conclusiones}

La realización de mediciones en el medio marino requiere un equipo con un alto grado de sofisticación tecnológica y por ende su adquisición resulta onerosa. Los gastos de importación representan en general, un porcentaje importante del costo del equipo. El riesgo de pérdida, cuando el equipo opera en aguas costeras durante la evolución de eventos hidrometeorológicos o en aquellas regiones donde las actividades pesqueras son importantes, es alta. En consecuencia, el mantenimiento y cuidado del equipo de medición es una actividad primordial en toda actividad de investigación limnológica y oceanográfica.

El presente trabajo contribuye con el diseño y construcción de una herramienta auxiliar que facilita las labores de localización y recuperación de equipo oceanográfico fondeado en aguas someras, con que se pretende disminuir el riesgo de pérdida. La herramienta auxiliar es el primer prototipo de un par de transceptores capaces de detectar frecuencias de $26 \mathrm{kHz}$ hasta profundidades ligeramente mayores de $30 \mathrm{~m}$ a través de un protocolo de aplicación de diseño propio para la transmisión-recepción de señales ultrasónicas. El costo de los transceptores es $40 \%$ menor al costo de equipo comercial de manufactura extranjera. Las pruebas operativas efectuadas al sistema ultrasónico demostraron que es posible calcular la distancia entre ambos transceptores con un error del orden de $\pm 0.4 \mathrm{~m}$, un buen lo- 
gro respecto al error de precisión de ubicación de los GPS comerciales. Este logro se debe en gran medida a no utilizar la velocidad del sonido promedio del océano. La velocidad del sonido en las zonas estuarinas y áreas costeras adyacentes es variable, caracterizarla, requiere al menos un año de mediciones con una frecuencia mensual en toda el área de interés. La obtención de mediciones confiables es por tanto cara, peligrosa dependiendo de las condiciones de la mar y lenta en términos del tiempo total, pero relativamente rápida en cuanto a la duración de un muestreo mensual. A pesar del largo tiempo que precisa obtener resultados en el medio marino, el trabajo de campo y el procesamiento de datos son de naturaleza práctica, es la manera correcta de trabajar en un sustrato que está en movimiento continuo.

En México, no existe una industria dedicada al diseño y fabricación de equipo oceanográfico aún cuando se tiene la necesidad de generar tecnología propia para la explotación de hidrocarburos en mar profundo, energía eléctrica mediante fuentes de energía oceánica, así como para atender las necesidades de la industria en el monitoreo de vertimientos en áreas fluviales y costeras. Por ello es importante impulsar los desarrollos tecnológicos. Este trabajo avanzó un paso pequeño para el logro de tal fin.

\section{Agradecimientos}

El apoyo financiero para la realización del presente trabajo fue gracias a los proyectos SIP-20120616 y CONACyT-FOMIX Tamaulipas 175692. Los comentarios de dos revisores anónimos ayudaron a enriquecer el manuscrito original.

\section{Referencias}

Chereskin T. y Howe B. Oceanographic measurements, en: Tropea C., Yarin A.L., Foss J.F. Springer handbook of experimental fluid mechanics, Berlin, Springer, 2007, pp. 1179-1217.

Farcas A., Thompson P.M., Merchant N.D. Underwater noise modelling for environmental impact assessment. Environmental Impact Assessment Review, volumen 57 (número 2): 114-122, 2016.

Hernández-Reyes A.I. Diseño y construcción de un transmisor y receptor acústico para la localización de equipo oceanográfico fondeado en un cuerpo de agua, (tesis de maestría en tecnología avanzada), México, Instituto Politécnico Nacional, 2015, 220 pp.

Instituto Nacional de la Pesca (INP). Catálogo de los sistemas de captura de las principales pesquerías comerciales, Secretaría de Medio Ambiente y Recursos Naturales-Dirección General de Investigación y Desarrollo Tecnológico Pesquero, México, Distrito Federal, 2000, 139 p.

Joseph A. Measuring ocean currents: tools, technologies, and data, San Diego, Elsevier, 2014, pp. 267-379.

Kleeman L. y Kuc R. Sonar sensing, en: Siciliano B. y Khatib O. Springer Handbook of Robotics, Berlin, Springer, 2008, pp. 491519.

Madry S. Global navigation satellite systems and their applications, Nueva York, Springer, 2015, pp. 27-55.

MacDonald I.T. y Mullarney J.C. A novel "FlocDrifter" platform for observing flocculation and turbulence processes in a lagrangian frame of reference. Journal of Atmospheric and Oceanic Technology, volumen 32 (número 3): 547-561, 2015.

Medwin H. Sounds in the sea: from ocean acoustics to acoustical oceanography, Cambridge, Cambridge University Press, 2005, pp. 105-113.

Murphy K., Heery B., Sullivan T., Zhang D., Paludetti L., Tong Lau K., Diamond D., Costa E., O'Connor N., Regan F. A lowcost autonomous optical sensor for water quality monitoring. Talanta, volumen 132 (número 1): 520-527, 2015.

Trask R. y Weller R. Moorings, en: Steele J.H., Thorpe S.A., Turekian K. Encyclopedia of Ocean Sciences, London, Academic Press, 2001, pp. 1850-1860.

Ulloa M.J. Interpreting underwater acoustic images of the upper ocean boundary layer. European Journal of Physics, volumen 18 (número 2): 301-309, 2007.

Waite A.D. Sonar for practicing engineers, Chichester, John Wiley \& Sons, 2005, pp. 50-56.

Williams R., Wright A.J., Ashe E., Blight L.K., Bruintjes R., Canessa R., Clark C.W., Cullis-Suzuki S., Dakin D.T., Erbe C., Hammond P.S., Merchant N.D., O'Hara P.D., Purser J., Radford A.N., Simpson S.D., Thomas L., Wale M.A. Impacts of anthropogenic noise on marine life: Publication patterns, new discoveries, and future directions in research and management. Ocean \& Coastal Management, volumen 115 (número 1): 17-24, 2015.

Vera M.D. Examples and applications in long-range ocean acoustics. European Journal of Physics, volumen 28 (número 6): 10631072, 2007.

Yellow Springs Instrument Company (YSI). CastAway ${ }^{\mathrm{TM}}$ CTD user's manual. YSI incorporated, Yellow Springs, 2010, 83 pp. 


\section{Este artículo se cita:}

\section{Citación estilo Chicago}

Hernández-Reyes, Alberto Isaac, Marco Julio Ulloa, Rogelio Ortega-Izaguirre. Un sistema ultrasónico de bajo costo para la localización de equipo oceanográfico en aguas someras. Ingeniería Investigación y Tecnología, XVII, 04 (2016): 511-520.

\section{Citación estilo ISO 690}

Hernandez-Reyes A.I., Ulloa M.J., Ortega-Izaguirre R. Un sistema ultrasónico de bajo costo para la localización de equipo oceanográfico en aguas someras. Ingeniería Investigación y Tecnología, volumen XVII (número 4), octubre-diciembre 2016: 511-520.

\section{Semblanzas de los autores}

Alberto Isaac Hernandez-Reyes. Es ingeniero electrónico por el Instituto Tecnológico de Ciudad Madero (2009), obtuvo el grado de maestro en tecnología avanzada por el Centro de Investigación en Ciencia Aplicada y Tecnología Avanzada, Unidad Altamira, del Instituto Politécnico Nacional (2015). Actualmente se desempeña como profesionista en el sector de la instrumentación electrónica y es aspirante al programa de doctorado en tecnología avanzada en la institución donde se graduó como maestro en tecnología avanzada.

Marco Julio Ulloa. Es oceanólogo por la Universidad Autónoma de Baja California (1989), maestro en ciencias con especialidad en oceanografía física por el Centro de Investigación Científica y de Educación Superior de Ensenada (CICESE, 1993) y doctor en filosofía por la Universidad de Southampton (2004). Realizó estudios de posdoctorado en el CICESE (2005). Actualmente se desempeña como profesor titular en el Centro de Investigación en Ciencia Aplicada y Tecnología Avanzada, Unidad Altamira, del Instituto Politécnico Nacional.

Rogelio Ortega-Izaguirre. Es ingeniero en sistemas computacionales por el Instituto Tecnológico de Ciudad Madero (1997), maestro en ciencias con especialidad en cómputo distribuido y paralelo por el Centro Nacional de Investigación y Desarrollo Tecnológico (2000) y doctor en tecnología avanzada por el Centro de Investigación en Ciencia Aplicada y Tecnología Avanzada, Unidad Altamira (2010). Desde el año 2011, se desempeña como profesor en el Centro de Investigación en Ciencia Aplicada y Tecnología Avanzada, Unidad Altamira, del Instituto Politécnico Nacional. 